\title{
Intermittent Hypoxic Episodes in Preterm Infants: Do They Matter?
}

\author{
Richard J. Martin Katherine Wang Özge Köroğlu Juliann Di Fiore Prabha Kc \\ Division of Neonatology, Rainbow Babies and Children's Hospital, Case Western Reserve University, \\ Cleveland, Ohio, USA
}

\section{Key Words}

Intermittent hypoxic episodes $\cdot$ Pulse oximetry $\cdot$ Extremely low birth weight infants

\begin{abstract}
Intermittent hypoxic episodes are typically a consequence of immature respiratory control and remain a troublesome challenge for the neonatologist. Furthermore, their frequency and magnitude are underestimated by clinically employed pulse oximeter settings. In extremely low birth weight infants the incidence of intermittent hypoxia progressively increases over the first 4 weeks of postnatal life, with a subsequent plateau followed by a slow decline beginning at weeks 6-8. Such episodic hypoxia/reoxygenation has the potential to sustain a proinflammatory cascade with resultant multisystem morbidity. This morbidity includes retinopathy of prematurity and impaired growth, as well as possible longer-term cardiorespiratory instability and poor neurodevelopmental outcome. Therapeutic approaches for intermittent hypoxic episodes comprise determination of optimal baseline saturation and careful titration of supplemental inspired oxygen, as well as xanthine therapy to prevent apnea of prematurity. In conclusion, characterization of the pathophysiologic basis for such intermittent hypoxic episodes and their consequences during early life is necessary to provide an evidence-based approach to their management.

Copyright $\odot 2011$ S. Karger AG, Basel
\end{abstract}

\section{Introduction}

Since the advent of neonatal intensive care, respiratory instability and the need to stabilize oxygenation have been recognized as significant clinical problems. Immature respiratory control and resultant apnea or hypoventilation with accompanying desaturation appear to be the consequence of a premature transition from fetal to neonatal life. The magnitude of the problem has been aggravated with the increased survival of extremely low birth weight infants.

Intermittent hypoxic events are typically the consequence of a sequence of events triggered by cessation of respiratory neural output, or ineffective ventilation as can occur during mechanical ventilation [1-3]. Several studies have shown that excessive or persistent apnea and bradycardia are associated with longer-term neurodevelopmental problems [4]. However, a causal relationship is hard to document and the role of accompanying desaturation is unclear. A substantial problem has been the inability to quantify the incidence and magnitude of apneic episodes via standard impedance monitoring as this technique cannot document the obstructed inspiratory efforts that commonly prolong central respiratory pauses.

Pulse oximetry provides an opportunity to more precisely quantify the frequency and magnitude of episodic desaturation, which is the consequence of apnea most

\section{KARGER}

() 2011 S. Karger AG, Basel

Fax +41613061234

E-Mail karger@karger.ch

www.karger.com
Accessible online at:

www.karger.com/neo
Richard J. Martin, MD

Division of Neonatology, Rainbow Babies and Children's Hospital

Case Western Reserve University, 11100 Euclid Avenue, Suite 3100

Cleveland, OH 44106-6010 (USA)

Tel. +1 216844 3387, E-Mail rxm6@case.edu 


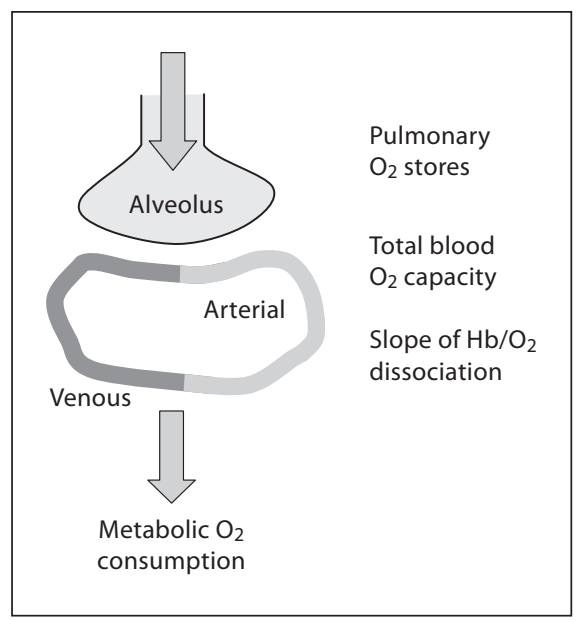

Fig. 1. Overview of the cardiorespiratory factors that influence the magnitude of oxygen desaturation during apnea of prematurity. Hypoxemia is likely enhanced by depletion of pulmonary oxygen stores at low lung volume, decreased blood oxygen carrying capacity, and increased peripheral oxygen consumption [5].

likely associated with short- or longer-term morbidity. It should be noted that several physiologic parameters contribute to the desaturation that accompanies cessation of ventilation. As indicated in figure 1, these include pulmonary oxygen stores that are a reflection of lung volume, total blood oxygen capacity comprising blood volume and hemoglobin content, as well as tissue oxygen consumption [5]. Nonetheless, pulse oximetry offers a technologically feasible, noninvasive technique for quantifying the relationship between intermittent hypoxic episodes and various pathophysiologic outcomes in preterm infants.

\section{Magnitude of the Problem}

The incidence of intermittent hypoxic events is presumably a function of maturation. To identify the natural history of intermittent hypoxic episodes, we followed a cohort of preterm infants over the first weeks of postnatal life. This required modification of the algorithm used for clinical pulse oximetry, as the latter typically employs a prolonged averaging time and low sample rate to minimize episodic desaturation rates and resultant monitor alarms [6]. Intermittent hypoxia (and hyperoxia) were documented using high-resolution pulse oximetry data (2-second sample rate and 2-second averaging time) recorded continuously from day 1 to 8 weeks' postnatal age.

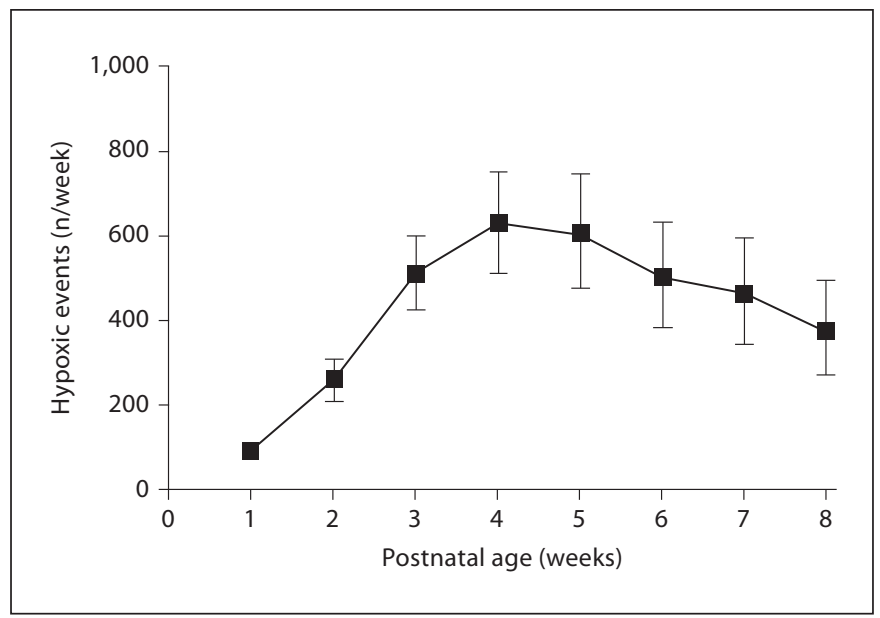

Fig. 2. Number of desaturation episodes [mean $(95 \% \mathrm{CI})]$ in a cohort of 24-28 weeks' gestation preterm infants over the first 8 weeks of life. There were relatively few desaturation episodes during the first week, followed by a progressive increase over weeks $2-4$, and then a decrease in weeks 6-8 [7].

Data were acquired using a pulse oximeter with a 2-second averaging time and a 2 -second sample rate (Radical; Masimo Corp., Irvine, Calif., USA). In this cohort of 79 infants (24-28 weeks' gestation) desaturation events, defined as a drop in arterial oxygen saturation $\left(\mathrm{SaO}_{2}\right)$ to $\leq 80 \%$ for between $10 \mathrm{~s}$ and $3 \mathrm{~min}$ duration, were identified using custom software (RTI, Research Triangle Park, N.C., USA). For each infant, the number of hypoxemic events was calculated for each week of postnatal age [7].

As seen in figure 2, there was a marked change in intermittent hypoxemic events over time with relatively few hypoxemic episodes occurring during the first week of life, then a progressive increase in weeks $2-4$, and finally a decrease in weeks $6-8$. These trends were apparent in the presence and absence of assisted ventilation, and consistent with historical beliefs that apnea of prematurity is less common in the first postnatal days. This may also be consistent with the decline in central chemosensitivity that is observed in neonatal rodent models during the first days of postnatal life [8]. The remarkably high incidence of intermittent hypoxic episodes in this population (e.g. 50-100/day) would likely be underestimated at conventional pulse oximeter settings employing prolonged averaging times. These data provide a basis for evaluating potential morbidity associated with such episodes, as addressed later in this review. 


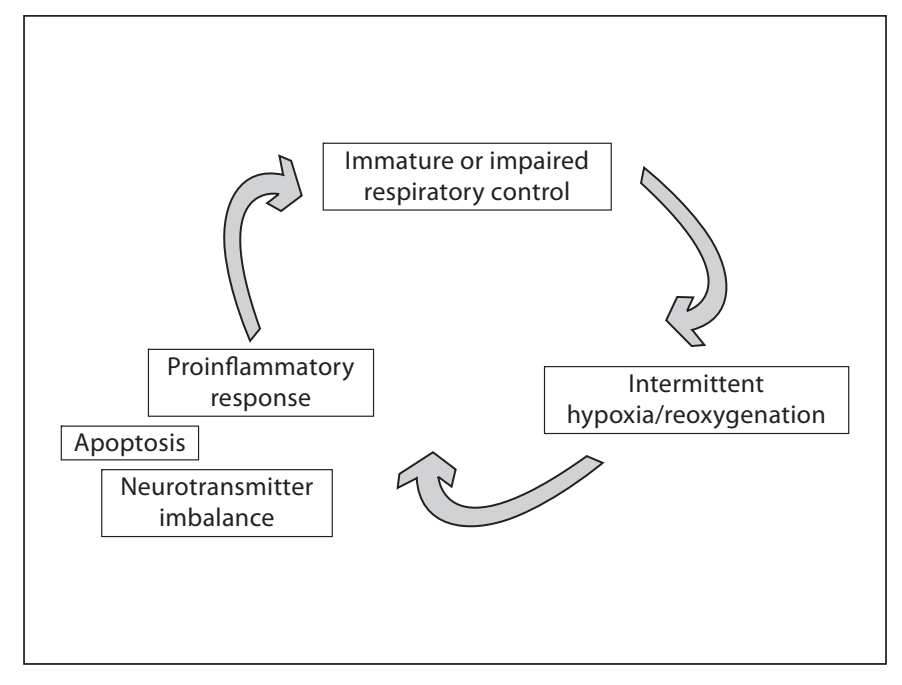

Fig. 3. Potentially vicious cycle created by immature respiratory control and resultant intermittent hypoxia.

\section{Intermittent Hypoxia as a Proinflammatory Stress}

Pre- and postnatal inflammation are widely implicated in both respiratory and neurodevelopmental morbidity associated with preterm birth. Antenatal exposure of the fetus to chorioamnionitis, and postnatal exposure to supplemental oxygen or baro/volutrauma have all been shown to elicit a proinflammatory response [9-12]. Although neonatal data are limited, there is evidence from other systems that intermittent hypoxia may also predispose to a proinflammatory cascade (fig. 3).

Much of our knowledge on this subject is derived from studies performed in adults with sleep apnea syndrome. Adult patients with obstructive sleep apnea syndrome have elevated TNF- $\alpha$ levels that fall after continuous positive airway pressure (CPAP) therapy [13]. Intermittent hypoxia, the hallmark of obstructive sleep apnea, results in activation of proinflammatory transcription factors such as NF- $\kappa$ B. These promote activation of various inflammatory cells, particularly lymphocytes and monocytes, with the downstream consequence of expression of proinflammatory mediators that may lead to diverse pathophysiology [14]. This novel line of investigation is worthy of study in a developmental model of cardiorespiratory disease.

Intermittent hypoxia is invariably associated with recovery to normoxia or even hyperoxia when recovery is associated with administration of supplemental oxygen. This, in turn, may represent an intermittent oxidant stress.

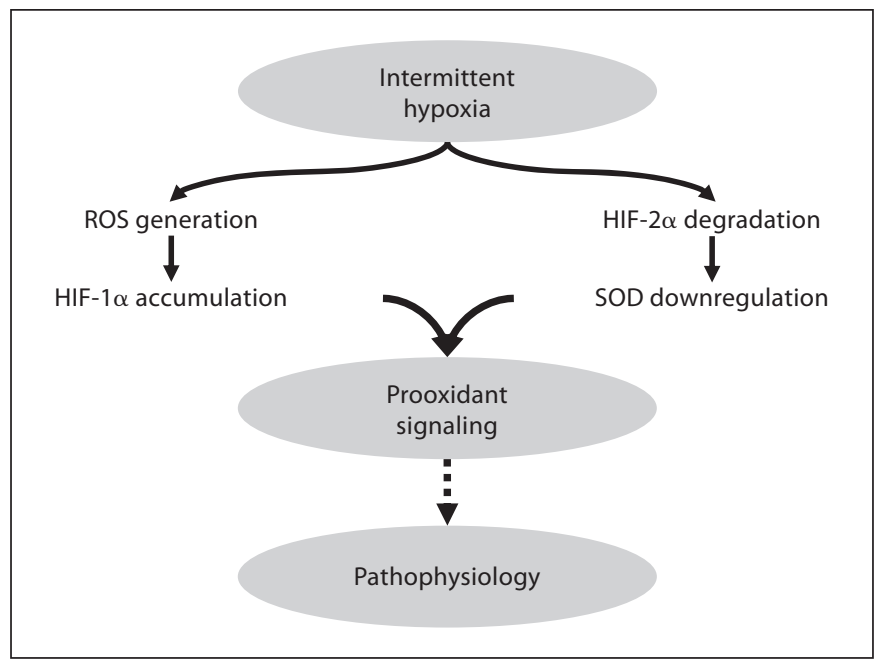

Fig. 4. The role of intermittent hypoxia and resultant generation of reactive oxygen species (ROS) in mediating expression of HIFs and the downstream balance of oxidant and antioxidant pathways [16]. SOD = Superoxide dismutase.

Once again, neonatal data are limited. Recent data in healthy adult volunteers have demonstrated that chronic intermittent hypoxia increases oxidative stress (e.g. DNA oxidation) by increasing production of reactive oxygen species without a compensatory increase in antioxidant activity [15]. Hypoxia-inducible factors (HIFs) mediate transcriptional responses to low oxygen. In both in vivo and in vitro models, HIF-1 $\alpha$ mediates some of the physiologic responses evoked by intermittent hypoxia. This may be compounded by intermittent hypoxia-induced degradation of HIF-2 $\alpha$ with resultant inhibition of transcription for the antioxidant enzyme superoxide dismutase [16] (fig. 4). A significant challenge in future studies is, therefore, to differentiate potential pathophysiologic effects of intermittent hypoxia in early life from the effects of oxidative stress associated with recovery to normoxia or hyperoxia.

Recent data in neonatal animal models demonstrate that postnatal exposure to a proinflammatory stimulus, in turn, inhibits central respiratory neural output, thus completing the vicious cycle proposed in figure 3 . We have recently shown that rat pups exposed to intratracheal lipopolysaccharide demonstrate increased expression of mRNA for the proinflammatory cytokines IL- $1 \beta$ and IL- 6 in the brainstem. This is associated with an attenuated ventilatory response to hypoxia, both before and after carotid sinus nerve transection [17]. This is consistent with prior data that endotoxemia impairs the ventilatory response to hypoxia in rat pups [18], and that prostaglandin-mediated 
Fig. 5. Proposed morbidities attributable to intermittent hypoxia in early life [57].

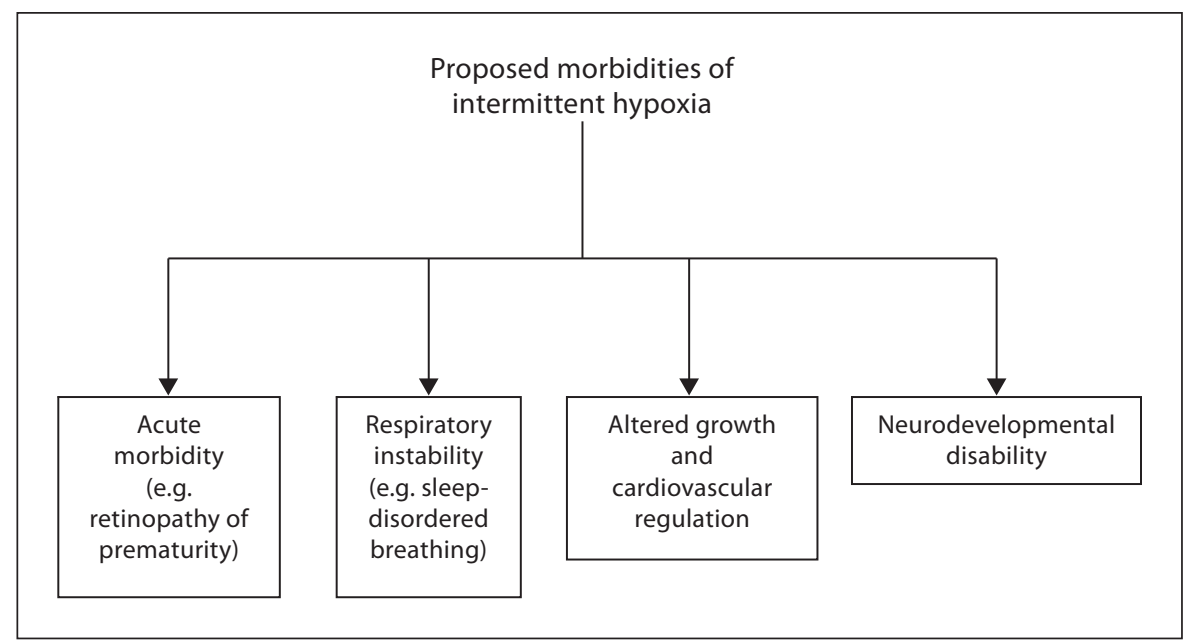

respiratory inhibition may be implicated [19]. In summary, as seen in figures 3 and 4 , we speculate that immature or impaired respiratory control and resultant intermittent hypoxia may initiate a proinflammatory and/or prooxidant cascade that, in turn, may further inhibit respiratory neural output and aggravate the problem. Furthermore, intermittent hypoxia may result in cellular changes such as apoptosis in neural structures and possible longer-lasting neurochemical alterations in animal models $[20,21]$. A variety of pathophysiologic problems may be a consequence of this sequence of events (fig. 5).

\section{Proposed Morbidity Related to Neonatal Intermittent Hypoxia}

\section{Retinopathy of Prematurity}

Retinopathy of prematurity (ROP) is a consequence of perturbations in retinal vascular development triggered by multiple factors, including oxygenation level. During the first phase of ROP, hyperoxia suppresses normal retinal vascularization, largely via inhibition of vascular endothelial growth factor. The second phase of ROP comprises retinal vascular overproliferation triggered in part by hypoxia-induced elevation of vascular endothelial growth factor and other growth factors [22]. Fluctuations in oxygenation have been previously implicated in the development of ROP, based primarily on intermittent arterial sampling. This provided only limited evaluation of the time course and frequency of oxygen desaturation in relation to ROP [23].

We have now documented a significant association between intermittent hypoxic episodes and the development of severe ROP requiring laser therapy [7] (fig. 6). This occurred in the cohort of infants of 24-28 weeks' gestation in whom high-resolution oxygen saturation was continuously monitored, as described earlier. After adjusting for confounding covariates, there were significant differences in the mean profiles of intermittent hypoxic events between laser-treated infants and those who had no ROP or did not require laser treatment. Thus, while hyperoxia is the most prominent risk factor for ROP, our data support the belief that fluctuations in oxygen saturation represent a significant confounding component. While we cannot exclude a contribution from iatrogenic hyperoxia secondary to a response to desaturation, the vast majority of hypoxic events did not meet our nursing criteria for intervention with supplemental oxygen. We are currently evaluating the patterns of oxygen fluctuation that are associated with ROP in an expanded cohort of preterm infants.

\section{Sleep-Disordered Breathing}

Sleep-disordered breathing is increasingly recognized as a potential source of developmental and behavioral problems in the pediatric population, although the role of intermittent hypoxia in these problems is controversial $[24,25]$. Two studies have demonstrated that preterm infants are at increased risk for sleep-disordered breathing in childhood and young adulthood $[26,27]$. The mechanisms underlying this relationship between preterm birth and later sleep-disordered breathing are unknown. Prior exposure to xanthines in the neonatal period appeared to increase the later risk, suggesting that apnea of prematurity and resultant intermittent hypoxia in early life may serve as a risk factor for later perturbations of sleep and breathing [28]. 
Physiologic studies in rat pups also lend support to the hypothesis that early onset intermittent hypoxia is associated with longer-lasting alteration in respiratory control $[29,30]$. Unfortunately, comparison of results between studies is confounded by the use of different intermittent hypoxia exposure protocols. Of particular note is the observation that neonatal rat pups exhibited a marked and sustained increase in sensory activity of the carotid body after chronic intermittent hypoxic exposure [31]. Reactive oxygen species have been implicated in these findings. Such an increase in carotid body excitation in response to hypoxic exposure may destabilize breathing, as has been shown in human preterm infant studies [32, 33]. Future study should focus on the possible relationship between neonatal intermittent hypoxia, subsequent hyperoxia, and the genesis of unstable breathing patterns in later life.

\section{Growth and Cardiovascular Consequences}

It is possible that postnatal intermittent hypoxic exposure has broader implications for growth trajectory and cardiovascular control beyond the neonatal period, although available data are limited. Earlier data from the UK proposed that reduced fetal growth, presumably due to an adverse fetal environment, predisposed to later cardiovascular disease [34]. Several subsequent studies in neonatal animal models employing various postnatal hypoxia protocols have demonstrated effects on postnatal weight gain and cardiovascular regulation [35-37]. A common feature of many of these studies is that intermittent versus sustained hypoxic exposures result in very different effects.

Short-term circulatory dysfunction, comprising a diminished pressor and heart rate response to stress, has been described in preterm infants, especially those with bronchopulmonary dysplasia. This has been attributed to prior hypoxic exposure in this population [38]. The effects of oxygen fluctuation may depend on pattern, magnitude, and duration of hypoxia-reoxygenation. For example, clustered versus dispersed episodes of hypoxia in early life appear to enhance the risk of ROP. Our own recent preliminary data demonstrate that neonatal rat pups exposed to 7 days of either clustered or dispersed intermittent hypoxia exhibit a transiently impaired growth trajectory with catch-up to normoxia controls by 4 weeks of age. The rat pups exposed to clustered hypoxia showed a lower arterial pressure as compared to normoxic controls, and both hypoxia-exposed groups had a lower baseline heart rate during follow-up [39]. Therefore, we believe the potential longer-term systemic implications of postnatal intermittent hypoxic exposure are worthy of further study (fig. 7).

Intermittent Hypoxic Episodes in Preterm Infants: Do They Matter?

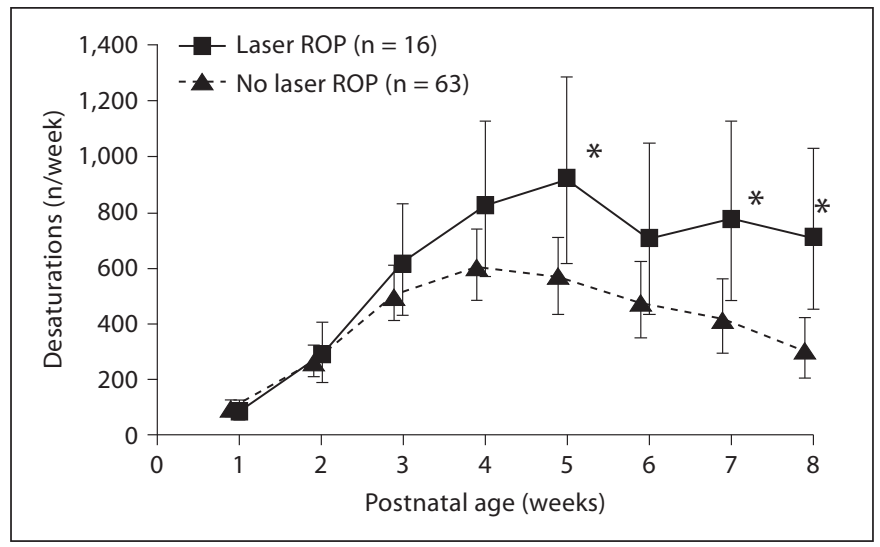

Fig. 6. Model-based estimate of intermittent hypoxemic events in preterm infants needing laser therapy for ROP vs. those who had no ROP or did not need laser therapy. Data [mean (95\% CI)] are controlled for gestational age, race, sex, multiple births, and SNAPPE-II score. There was an overall higher incidence of hypoxemic events in the laser-treated infants with significant differences at 5,7 , and 8 weeks of age $\left({ }^{*} p<0.05\right)[7]$.

\section{Neurodevelopmental Outcome}

Several studies have attempted to correlate the incidence and duration of apnea of prematurity with later neurodevelopmental outcome. The problem of correlating apnea with outcome is enhanced by the fact that nursing reports of apnea severity may be unreliable, and impedance monitoring techniques will fail to identify mixed and obstructive events. Despite these limitations, available data suggest a link between the number of days of apnea plus the number of days of assisted ventilation, and impaired neurodevelopmental outcome [40]. A relationship has also been shown between delay in resolution of apnea and bradycardia beyond 36 weeks' corrected age and a higher incidence of unfavorable neurodevelopmental outcome [4]. Future studies might better focus on the incidence and severity of desaturation events, as the technical ability to do this is now available, and it is likely that recurrent hypoxia is the detrimental feature of the immature respiratory control exhibited by preterm infants. Of course the problem remains that a definitive relationship between intermittent hypoxia and later outcome may be associative rather than causal, although controlling for confounding factors may resolve this challenge. Studies in animal models may also be helpful. For example, neurofunctional handicap in neonatal mice with hyperoxia-induced lung injury was exacerbated by exposure to intermittent hypoxia [41].

Neonatology 2011;100:303-310 


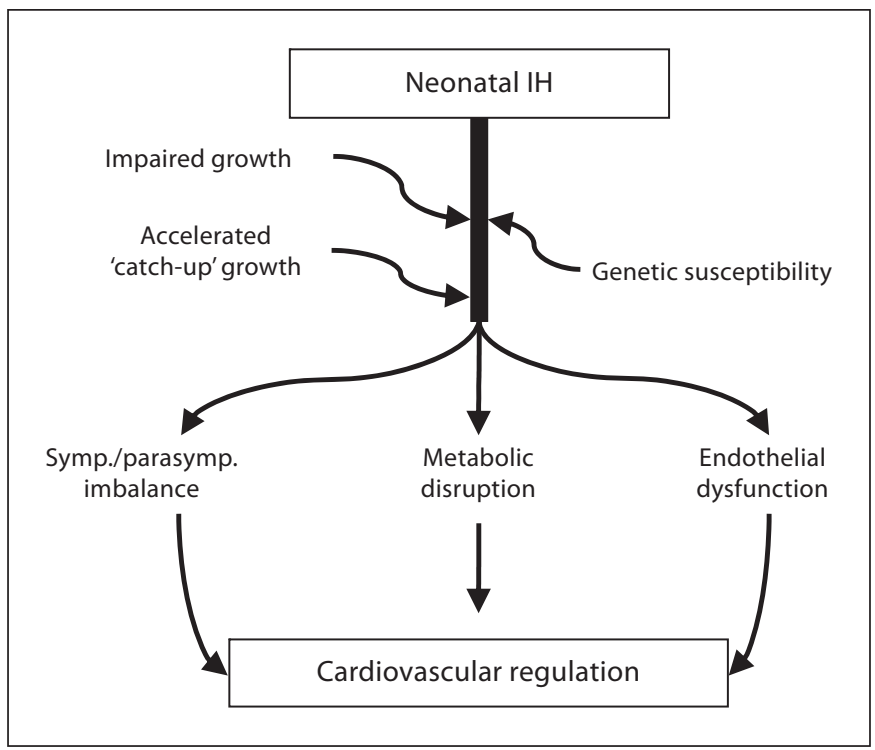

Fig. 7. Potential broad implications of neonatal intermittent hypoxia $(\mathrm{IH})$ on growth patterns and longer-term cardiovascular and metabolic regulation.

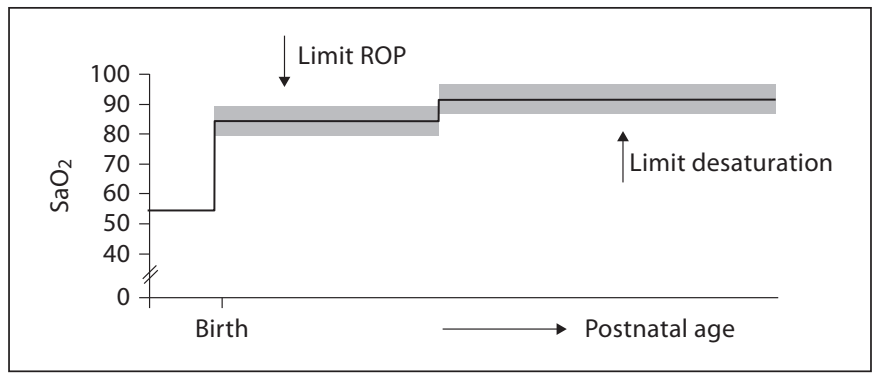

Fig. 8. Possible future guidelines for optimizing baseline oxygen saturation in preterm infants and minimizing morbidity. Clearly such an approach needs careful future study before being considered in clinical practice.

\section{Therapeutic Approaches to Prevent Intermittent Hypoxia}

\section{Optimizing Baseline Oxygen Saturation}

It has long been suspected that targeting a lower baseline oxygen saturation in infants with bronchopulmonary dysplasia results in more desaturation [42]. Meanwhile, multiple large trials, some of which are ongoing, have randomized infants to two different levels of baseline oxygen saturation in order to identify resultant morbidity. Based on a need for prolonged oxygen supplementation when levels of $95 \%$ were targeted, the current focus is on $85-89 \%$ versus $90-95 \%$ oxygen in preterm infants $<28$ weeks' gestation. While the lower targeted range is associated with less ROP, there appears to be a slightly, but significantly, higher mortality in this group [43, 44]. In a subgroup of infants from the latter trial, we have preliminarily identified a higher incidence of intermittent hypoxic episodes in the low oxygen targeted group [45]. It is tempting to speculate that the resultant intermittent hypoxia and/or oxidative stress from these desaturation episodes may predispose to this adverse mortality outcome. An additional speculation is that high-risk preterm infants may need to be subjected to a stepwise increase in baseline oxygen during their postnatal course as was proposed in the STOP-ROP Trial [46], and as indicated in figure 8. Clearly, this approach needs careful further consideration for optimal target levels of baseline oxygenation, and for timing of any stepwise change in targeted oxygenation.

\section{Automated Control of Inspired Oxygen}

Preterm infants receiving mechanical ventilation frequently have substantial fluctuations in oxygen saturation. These episodes of desaturation are a real challenge to personnel trying to maintain baseline oxygen saturation in a predetermined range. Automated adjustments of inspired oxygen would have the potential to reduce the time-consuming task of repetitively changing supplemental oxygen. This automated technique has now been compared to routine adjustments of inspired oxygen as performed by clinical personnel in infants of 24-27 weeks' gestation [47]. During the automated period, time with oxygen saturation within the intended range of 8793\% increased significantly and times in the hyperoxic range were significantly reduced. This was not associated with a clear benefit for hypoxic episodes. While times with an oxygen saturation $<80 \%$ did not differ, the automated system actually increased time with oxygen saturation between 80 and $86 \%$. Therefore, this novel technique may not be an obvious solution to minimize episodic intermittent hypoxia.

\section{Xanthine Therapy}

Given that apnea is the major cause of intermittent hypoxic episodes in preterm infants, CPAP and xanthine therapy should be the major therapeutic approaches. CPAP is a safe and effective proven therapy that serves to maintain functional residual capacity and resultant oxygenation as well as splinting the upper airway and preventing its closure in the face of decreased respiratory drive. Xanthine therapy has been used to prevent and 
treat apnea of prematurity since the 1970s. Its primary mechanism of action in the perinatal period is thought to be blockade of inhibitory adenosine $\mathrm{A}_{1}$ receptors with resultant excitation of respiratory neural output [48]. An alternative mechanism of caffeine action is blockade of excitatory adenosine $A_{2 A}$ receptors at GABAergic neurons and resultant decrease in GABA output, resulting in excitation of respiratory neural output [49].

These complex neurotransmitter interactions elicited by caffeine led to concerns regarding its safety and a large multicenter trial was undertaken in the 1990s. The results of this study have demonstrated that caffeine treatment is effective in decreasing the rate of bronchopulmonary dysplasia and improving neurodevelopmental outcome at 18-21 months, especially in those receiving respiratory support $[50,51]$. It is possible that this benefit is secondary to decrease in apnea and resultant intermittent hypoxic episodes; however, this is speculative. A small earlier study actually did not demonstrate a decrease in hypoxic episodes, as measured by transcutaneous $\mathrm{PO}_{2}$, in response to xanthine therapy [52]. Xanthine therapy may also play a role in modulating inflammatory mechanisms in preterm infants as demonstrated in cord blood monocytes $[53,54]$. As indicated earlier, relief of both intermittent hypoxia and proinflammatory mechanisms may be interrelated mechanisms. The clinical benefits of xanthine therapy in preterm infants should trigger interest in a 'bedside-to-bench' approach to enhance our understanding of underlying mechanisms.

\section{Conclusion}

Despite the sophistication of modern neonatal intensive care, many basic pathophysiologic questions remain to be addressed. While the frequency of intermittent episodes of hypoxia or desaturation has been identified, it is unclear whether they represent a source of acute or chronic morbidity. It is even possible that intermittent hypoxic events in early life may provide later benefit by way of oxygen tolerance in humans, as has been proposed in rodent studies $[55,56]$. There is widespread agreement that apnea and ineffective ventilation are the main precipitants of intermittent hypoxic events. It is possible that episodic hypoxia/reoxygenation establishes or promotes a proinflammatory/prooxidant cascade with adverse effects on multiple organ systems. Greater understanding of the underlying pathophysiology is needed in order to provide a rationale for preventing or treating these episodes with either targeted supplemental oxygen or aggressive pharmacotherapy in the form of xanthine treatment.

\section{Acknowledgment} (P.K.)

Supported by NIH grants HL 98628 (R.J.M.) and HL 87620

\section{Disclosure Statement}

None of the authors has a conflict of interest.

\section{References}

1 Abu-Shaweesh JM, Martin RJ: Neonatal apnea: what's new? Pediatr Pulmonol 2008;43: 937-944.

2 Dimaguila MA, Di Fiore JM, Martin RJ, Miller MJ: Characteristics of hypoxemic episodes in very low birth weight infants on ventilatory support. J Pediatr 1997;130:577583.

$\checkmark 3$ Esquer C, Claure N, D’Ugard C, Wada Y, Bancalari E: Role of abdominal muscles activity on duration and severity of hypoxemia episodes in mechanically ventilated preterm infants. Neonatology 2007;92:182-186.

4 Pillekamp F, Hermann C, Keller T, et al: Factors influencing apnea and bradycardia of prematurity - implications for neurodevelopment. Neonatology 2007;91:155-161.

5 Sands SA, Edwards BA, Kelly VJ, Davidson MR, Wilkinson MH, Berger PJ: A model analysis of arterial oxygen desaturation during apnea in preterm infants. PLoS Comput Biol 2009;5:e1000588.
6 Ahmed SJ, Rich W, Finer NN: The effect of averaging time on oximetry values in the premature infant. Pediatrics 2010;125:e115e121.

7 Di Fiore JM BJ, Orge F, Schutt A, Schluchter M, Cheruvu VK, Walsh M, Finer N, Martin RJ: A higher incidence of intermittent hypoxemic episodes is associated with severe retinopathy of prematurity. J Pediatr 2010; 157:69-73

8 Darnall RA: The role of $\mathrm{CO}_{2}$ and central chemoreception in the control of breathing in the fetus and the neonate. Resp Physiol Neurobiol 2010;173:201-212.

-9 Kallapur SG, Nitsos I, Moss TJ, et al: IL-1 mediates pulmonary and systemic inflammatory responses to chorioamnionitis induced by lipopolysaccharide. Am J Resp Crit Care Med 2009;179:955-961.
10 Leviton A, Allred EN, Kuban KC, et al: Microbiologic and histologic characteristics of the extremely preterm infant's placenta predict white matter damage and later cerebral palsy. The ELGAN Study. Pediatr Res 2010; 67:95-101.

11 Speer CP: Chorioamnionitis, postnatal factors and proinflammatory response in the pathogenetic sequence of bronchopulmonary dysplasia. Neonatology 2009:95:353-361.

12 Thomas W, Speer CP: Chorioamnionitis: important risk factor or innocent bystander for neonatal outcome? Neonatology 2011;99: 177-187.

13 Ryan S, Taylor CT, McNicholas WT: Selective activation of inflammatory pathways by intermittent hypoxia in obstructive sleep apnea syndrome. Circulation 2005; 112:26602667.

$\checkmark 14$ Mehra R, Redline S: Sleep apnea: a proinflammatory disorder that coaggregates with obesity. J Allergy Clin Immunol 2008;121: 1096-1102. 
15 Pialoux V, Hanly PJ, Foster GE, et al: Effects of exposure to intermittent hypoxia on oxidative stress and acute hypoxic ventilatory response in humans. Am J Resp Crit Care Med 2009;180:1002-1009.

16 Nanduri J, Wang N, Yuan G, et al: Intermittent hypoxia degrades HIF-2alpha via calpains resulting in oxidative stress: implications for recurrent apnea-induced morbidities. Proc Natl Acad Sci USA 2009;106: 1199-1204

17 Balan KV, Kc P, Hoxha Z, Mayer CA, Wilson CG, Martin RJ: Vagal afferents modulate cytokine-mediated respiratory control at the neonatal medulla oblongata. Resp Physiol Neuro Biol 2011, E-pub ahead of print.

18 Ladino JBE, Suguihara C: Ventilatory response to hypoxia during endotoxemia in young rats: role of nitric oxide. Pediatr Res 2007:62:134-138.

19 Olsson A, Kayhan G, Lagercrantz H, Herlenius E: IL-1 beta depresses respiration and anoxic survival via a prostaglandin-dependent pathway in neonatal rats. Pediatr Res 2003;54:326-331.

20 Gozal D, Daniel JM, Dohanich GP: Behavioral and anatomical correlates of chronic episodic hypoxia during sleep in the rat. J Neurosci 2001;21:2442-2450.

21 Decker MJ, Hue GE, Caudle WM, et al: Episodic neonatal hypoxia evokes executive dysfunction and regionally specific alterations in markers of dopamine signaling. Neuroscience 2003;117:417-425.

22 Chen J, Smith LE: Retinopathy of prematurity. Angiogenesis 2007;10:133-140.

-23 York JR, Landers S, Kirby RS, Arbogast PG, Penn JS: Arterial oxygen fluctuation and retinopathy of prematurity in very-low-birthweight infants. J Perinatol 2004;24:82-87.

24 Urschitz MS, Eitner S, Guenther A, et al: Habitual snoring, intermittent hypoxia, and impaired behavior in primary school children. Pediatrics 2004;114:1041-1048.

25 Gottlieb DJ, Chase C, Vezina RM, et al: Sleep-disordered breathing symptoms are associated with poorer cognitive function in 5-year-old children. J Pediatr 2004;145:458464.

26 Rosen CL, Larkin EK, Kirchner HL, et al: Prevalence and risk factors for sleep-disordered breathing in 8- to 11-year-old children: association with race and prematurity. J Pediatr 2003;142:383-389.

-27 Paavonen EJ, Strang-Karlsson S, Raikkonen $\mathrm{K}$, et al: Very low birth weight increases risk for sleep-disordered breathing in young adulthood: the Helsinki Study of Very Low Birth Weight Adults. Pediatrics 2007;120: 778-784.

28 Hibbs AM, Johnson NL, Rosen CL, et al: Prenatal and neonatal risk factors for sleep disordered breathing in school-aged children born preterm. J Pediatr 2008;153:176182.
29 Reeves SR, Mitchell GS, Gozal D: Early postnatal chronic intermittent hypoxia modifies hypoxic respiratory responses and long-term phrenic facilitation in adult rats. Am J Physiol 2006;290:R1664-R1671.

30 Julien C, Bairam A, Joseph V: Chronic intermittent hypoxia reduces ventilatory longterm facilitation and enhances apnea frequency in newborn rats. Am J Physiol 2008; 294:R1356-R1366.

- 31 Pawar A, Peng YJ, Jacono FJ, Prabhakar NR: Comparative analysis of neonatal and adult rat carotid body responses to chronic intermittent hypoxia. J Appl Physiol 2008;104: 1287-1294.

32 Nock ML, Di Fiore JM, Arko MK, Martin RJ: Relationship of the ventilatory response to hypoxia with neonatal apnea in preterm infants. J Pediatr 2004;144:291-295.

33 Cardot V, Chardon K, Tourneux P, et al: Ventilatory response to a hyperoxic test is related to the frequency of short apneic episodes in late preterm neonates. Pediatr Res 2007;62: 591-596.

34 Barker DJ, Osmond C, Golding J, Kuh D, Wadsworth ME: Growth in utero, blood pressure in childhood and adult life, and mortality from cardiovascular disease. BMJ 1989;298:564-567.

35 Souvannakitti D, Kumar GK, Fox A, Prabhakar NR: Neonatal intermittent hypoxia leads to long-lasting facilitation of acute hypoxiaevoked catecholamine secretion from rat chromaffin cells. J Neurophysiol 2009;101: 2837-2846.

36 Soukhova-O'Hare GK, Cheng ZJ, Roberts AM, Gozal D: Postnatal intermittent hypoxia alters baroreflex function in adult rats. Am J Physiol Heart Circ Physiol 2006;290: H1157-H1164

37 Farahani R, Kanaan A, Gavrialov O, et al: Differential effects of chronic intermittent and chronic constant hypoxia on postnatal growth and development. Pediatr Pulmonol 2008;43:20-28.

38 Cohen G, Lagercrantz H, Katz-Salamon M: Abnormal circulatory stress responses of preterm graduates. Pediatr Res 2007;61:329334.

39 Pozo M, Köroğlu ÖA, Litvin DG, Cave AT, Martin RJ, Di Fiore J, Kc P: Postnatal repetitive intermittent hypoxic exposure induces long term alteration in cardiovascular regulation. EPAS 2011:2920.283.

40 Janvier A, Khairy M, Kokkotis A, et al: Apnea is associated with neurodevelopmental impairment in very low birth weight infants. J Perinatol 2004:24:763-768.

41 Ratner V, Kishkurno SV, Slinko SK, et al: The contribution of intermittent hypoxemia to late neurological handicap in mice with hyperoxia-induced lung injury. Neonatology 2007;92:50-58.
42 McEvoy C, Durand M, Hewlett V: Episodes of spontaneous desaturations in infants with chronic lung disease at two different levels of oxygenation. Pediatr Pulmonol 1993;15: 140-144.

43 Carlo WA, Finer NN, Walsh MC, et al: Target ranges of oxygen saturation in extremely preterm infants. N Engl J Med 2010;362: 1959-1969.

44 Stenson B, Brocklehurst P, Tarnow-Mordi W: Increased 36-week survival with high oxygen saturation target in extremely preterm infants. N Engl J Med 2011;364:1680-1682.

45 Di Fiore J, et al: Low oxygen saturation target range is associated with increased incidence of intermittent hypoxemia. EPAS 2011: 3305.7.

46 The STOP-ROP Multicenter Study Group. Supplemental therapeutic oxygen for prethreshold retinopathy of prematurity (STOP-ROP), a randomized, controlled trial. I: Primary outcomes. Pediatrics 2000;105: 295-310.

-47 Claure N, Bancalari E, D'Ugard C, et al: Multicenter crossover study of automated control of inspired oxygen in ventilated preterm infants. Pediatrics 2011;127:e76-e83.

48 Herlenius E, Aden U, Tang LQ, Lagercrantz $\mathrm{H}$ : Perinatal respiratory control and its modulation by adenosine and caffeine in the rat. Pediatr Res 2002;51:4-12.

49 Mayer CA, Haxhiu MA, Martin RJ, Wilson CG: Adenosine $A_{2 A}$ receptors mediate GABAergic inhibition of respiration in immature rats. J Appl Physiol 2006;100:91-97.

50 Davis PG, Schmidt B, Roberts RS, et al: Caffeine for Apnea of Prematurity Trial: benefits may vary in subgroups. J Pediatr 2010;156: 382-387.

51 Schmidt B, Roberts RS, Davis P, et al: Longterm effects of caffeine therapy for apnea of prematurity. N Engl J Med 2007;357:18931902

52 Bucher HU, Duc G: Does caffeine prevent hypoxaemic episodes in premature infants? A randomized controlled trial. Eur J Pediatr 1988;147:288-291.

53 Chavez-Valdez R, Wills-Karp M, Ahlawat R, et al: Caffeine modulates TNF-alpha production by cord blood monocytes: the role of adenosine receptors. Pediatr Res 2009;65: 203-208.

54 Haskó G, Cronstein BN: Adenosine: an endogenous regulator of innate immunity. Trends Immunol 2004;25:33-39.

-55 Clark JM, Lambertsen CJ, Gelfand R, Troxel ALBL: Optimization of oxygen tolerance extension in rats by intermittent exposure. J Appl Physiol 2006;100:869-879.

56 Adle-Biassette $\mathrm{H}$, Bouslama M, Matrot B, Favrais G, Gallego J, Gressens P: Moderate intermittent hypoxia protects neurodevelopment in newborn mice. EPAS 2011: 4534.510.

57 Martin RJ, Wilson CG: What to do about apnea of prematurity? J Appl Physiol 2009; 107:1015-1016. 